\title{
COMPUTING TRIANGULATIONS OF THE CONVEX POLYGON IN PHP/MYSQL ENVIRONMENT
}

\author{
Sead H. Mašović, Muzafer H. Saračević, Predrag S. Stanimirović \\ and Predrag V. Krtolica
}

\begin{abstract}
In this paper we implement the Block method for convex polygon triangulation in the web environment (PHP/MySQL). Our main aim is to show the advantages of the usage of web technologies in performing complex algorithm from computer graphics. The basic assumption is that once obtained, the results can be stored in a database and used for other calculations. Databases are convenient and structured methods of sharing and retrieving data. We have performed a comparative analysis of the developed program with respect to two criteria: CPU time in generating triangulation and $\mathrm{CPU}$ time in reading results from the database.
\end{abstract}

Keywords: Computer graphics, Polygon triangulation, Block method, PHP/MySQL.

\section{Introduction and preliminaries}

Polygon triangulation is an important problem applicable in computer graphics. Restricted to the convex case, the decomposition of a polygon is done into triangles by a maximal set of non-intersecting diagonals.

Let $P_{n}$ denote a polygon with $n$ vertices. The total number $T_{n}$ of $n$-gon triangulations is

$$
T_{n}=C_{n-2}=\frac{1}{n-1}\left(\begin{array}{c}
2 n-4 \\
n-2
\end{array}\right)=\frac{(2 n-4) !}{(n-1) !(n-2) !}, \quad n \geqslant 3
$$

Here, $C_{n}$ represents the $n$th Catalan number (see e.g. [9]).

The set of all triangulations of the convex polygon $P_{n}$ is denoted by $\mathcal{T}_{n}$. Diagonal connecting vertices $i$ and $j$ are denoted by $\delta_{i, j}$. An outer face edge can be considered as a diagonal, while nonadjacent vertices are connected by an internal diagonal.

Received June 10, 2018; accepted January 29, 2019

2010 Mathematics Subject Classification. Primary 32B25; Secondary 68N15, 68P15 
Many authors deal with the problem of how to generate the triangulation of a convex polygon based on some criterion. In this paper we implement the Block method for convex polygon triangulation [6] in the web environment using $\mathrm{PH}-$ $\mathrm{P} /$ MySQL technologies.

The combination of PHP and MySQL is the most convenient approach to dynamic, database-driven web design application. Due to its open source roots, it is free to implement and is therefore an extremely popular option for web development.

PHP is extremely powerful and exceptionally fast it can run on even the most basic hardware, and it hardly puts a dent in the system resources. The main characteristics of PHP are described in [2].

According to the TIOBE Programming Community index ${ }^{1}$, the PHP programming language is one of the top 10 most popular programming languages. Eighty percent of the top 10 million websites use PHP in one way or the other, including Facebook and Wikipedia.

PHP, as a scripting language, is popular among web developers because of its ability to interact with database systems.

MySQL is probably the most popular database management system for web servers.

MySQL is a fast and powerful, yet easy-to-use, database system that offers just about anything a website would need in order to find and serve up data to browsers.

The combination of PHP and MySQL can be used to build simple or complex and high traffic websites (see for e.g. [1, 7]). Similarly, the authors [4] used PHP/MySQL environment for computing the weighted Moore-Penrose inverse employing the partitioning method, as well as for storing the generated results.

This paper is organized as follows. In the Section 2 we present the main parts of the Block method for convex polygon triangulation. In Section 3 we describe the implementation of the algorithm in the PHP/MySQL environment. Section 4 includes a comparative analysis of the obtained numerical results.

\section{Block method for convex polygon triangulation}

Here we restate the Block method for convex polygon triangulation [6] which is the subject of our implementation.

The general strategy of the method is to decompose the problem into smaller dependant subproblems. Each subproblem is solved only once and used many times avoiding unnecessary repetitions of calculation.

The method is based on the usage of the previously generated triangulations for polygon with a smaller number of vertices. More precisely, the algorithm generates the set $\mathcal{T}_{n}$ using all the previously generated triangulations $\mathcal{T}_{b}$, where $b<n$. The set $\mathcal{T}_{b}$ is used as many times as necessary as a block, i.e. it is repeated several times in $\mathcal{T}_{n}$.

\footnotetext{
${ }^{1}$ https://www.tiobe.com/tiobe-index/
} 
The formal statement of the subject method is given by the following equation

$$
T_{n}=2 T_{n-1}+\operatorname{rest}\left(R_{n}\right) .
$$

The general idea of the Block method uses $T_{n-1}$ to generate $T_{n}$, which is illustrated in Figure 2.1, where one case of the transformation process from a $P_{5}$ triangulation into two corresponding $P_{6}$ triangulations is presented. In part (a) we see that the diagonals $\delta_{2,4}$ and $\delta_{2,5}$ make all vertices closed except the vertices 1,2 , 5 , and 6 which form a quadrilateral. The parts (b) and (c) show two ways to triangulate a quadrilateral, which gives two $P_{6}$ triangulations having a $P_{5}$ triangulation as a starting block.

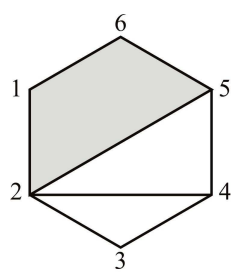

(A)

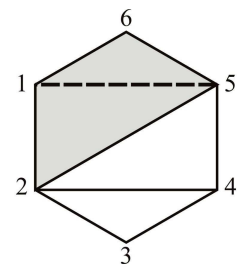

(B)

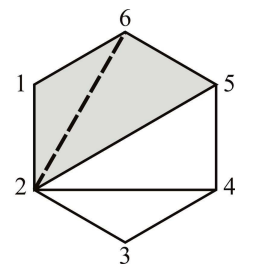

(C)

FIG. 2.1: Transformation from a $P_{5}$ into the corresponding $P_{6}$ triangulations. $P_{5}=\{(2,4),(2,5)\} \rightarrow P_{6}=\{(2,4),(2,5),(1,5) \&(2,4),(2,5),(2,6)\}$

Starting from the assumption that triangulation has at least two ears and that, in the worst case, one ear can be a vertex $n$, then we always have at least one ear among the rest of the vertices.

For the correctness of the algorithm in the procedure used for finding and eliminating closed vertices, the authors introduced a list of ordered pairs of the form

$$
L=\{(1,1),(2,2), \ldots,(n, n)\} .
$$

After the elimination of $n-l$ pairs the list $L$ becomes

$$
L=\left\{\left(s, i_{s}\right), s=1, \ldots, l\right\}, 4 \leq l \leq n, i_{l}=n .
$$

The values $i_{s}, s=1, \ldots, l$ are the vertex marks, while the values $1, \ldots, l$ represent the relative vertex positions in the list $L$.

Here we restate two additional algorithms 2.1 - Pair elimination \& 2.2 - Form a quadrilateral, which are part of the Block Method Algorithm 2.3. 
Algorithm 2.1 Pair elimination

Require: List $L$ of the form (2.3) and vertices $i_{p}$ and $i_{q}$, where $d(p, q)=2$.

1: Remove from the list $L$ the pair placed between the pairs $\left(p, i_{p}\right)$ and $\left(q, i_{q}\right)$ in a circular manner.

2: Decrease by one the first pair members in the pairs following the eliminated one.

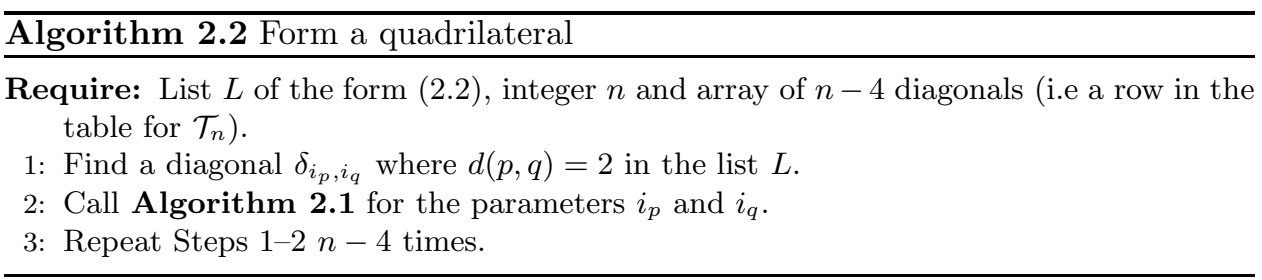

The main algorithm for the Block method is presented below.

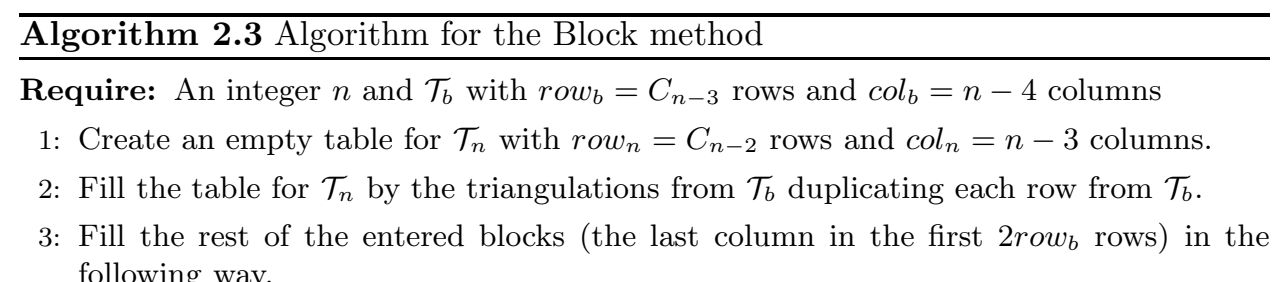
following way.

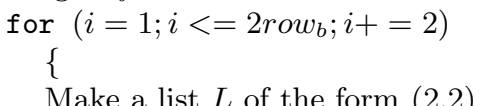

Call Algorithm 2.2 with row $i$ from the table for $\mathcal{T}_{n}$ as a parameter.

From the remaining four vertices in the list $L$ make a diagonal $\delta_{i_{1}, i_{3}}$ and place it in the last column of the row $i$ and diagonal $\delta_{i_{2}, i_{4}}$ and place it in the last column of the row $i+1$. \}

4: Fill the rest of the table for $\mathcal{T}_{n}$ containing $T_{n}-2 T_{b}$ rows.

4.1 Filling the first $n-4$ columns in the last row $_{n}-2$ row $_{b}$ rows.

$i=2 *$ row $_{b}+1$;

Make the list $L$ of the form (2.2).

Eliminate the vertices adjacent to $n$ calling Algorithm 2.1 for the parameters 1 and $n-1$.

Fill the current table row $i$ by diagonals $\delta_{2, n}, \delta_{3, n}, \ldots, \delta_{n-2, n}$.

The first $n-4$ columns in the rest rown -2 row $_{b}-1$ rows should be filled with the diagonals with the last vertex $n$, while the first vertices are combinations of the $(n-4)$ th class in the set $\{2,3, \ldots, n-2\}$. The number of these combinations is $\left(\begin{array}{l}n-3 \\ n-4\end{array}\right)=n-3$.

4.2 Filling the last column in the last $\left(\right.$ row $_{n}-2$ row $\left._{b}\right)$ rows.

for $\left(i=2\right.$ row $_{b}+2 ; i<=$ row $\left._{n} ; i++\right)$

\{

Make the list $L$ of the form (2.2). 
Call Algorithm 2.2 with the row $i$ from the table for $\mathcal{T}_{n}$ as a parameter. From the remaining four vertices in the list $L$ make a diagonal $\delta_{i_{1}, i_{3}}$ and place it in the last column of the row $i$.

\}

\section{PHP/MySQL implementation of Block method}

The most used architecture for development of web applications is three-tier architecture (Figure 3.1). Three-tier web architecture is a unique system for developing web database applications which work around the three-tier model comprising the database tier at the bottom, the application tier in the middle and the client tier on top.

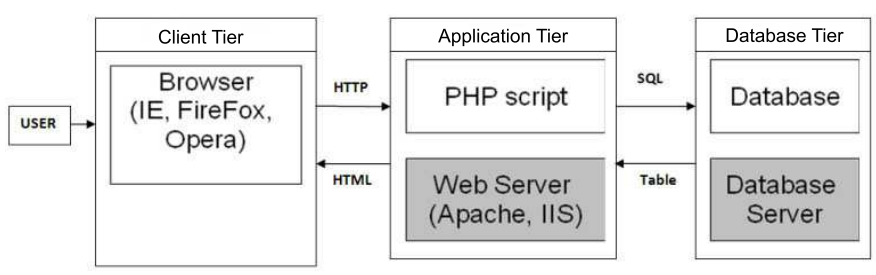

FIG. 3.1: Three-tier Web Architecture

The web interface of our application is given in Figure 3.2

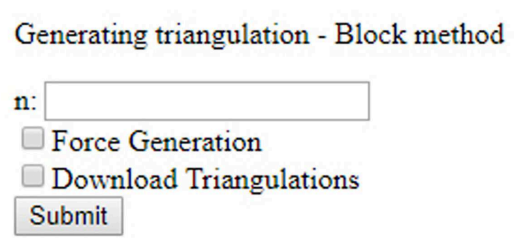

FiG. 3.2: Web interface of the application

According to the three-tier architecture, our application is organized as follows:

- On the client tier we have the web interface;

- Algorithm for the Block method is performed on application tier;

- Generated triangulations are stored on database tier;

In what follows, we presents a detailed view of the application scenario: 
First, we have to enter the value $n$ for which convex polygon we want to calculate triangulation.

Preconditions: $n \geq 4$

Second, when we press the submit button, Application search in database:

Case 1: Force Generation = Not marked

Have we already calculated triangulations of $n$ in the database;

- If we have, the application displays the results of $T_{n}$ in the browser;

- If we have not, the application checks if we have the results of $T_{n-1}$ in the database:

* If we have, then call Algorithm 2.3

* If we do not, the preconditions of Algorithm 2.3 are not fulfilled;

\section{Case 2: Force Generation = Marked}

Have we already calculated triangulations of $n-1$ in the database;

- If we have, then call Algorithm 2.3

- If we have not, the preconditions of Algorithm 2.3 are not fulfilled;

Third, the output results can be downloaded in a CSV format if we mark "Download Triangulation".

Example 3.1. Let us illustrate how the application works on generating hexagon triangulations using the already known pentagon triangulations.

First, $n=6$;

Preconditions fulfilled: $6 \geq 4$;

Second, the submit button is pressed

\section{Case 1: Force Generation = Not marked}

- The application checks if we have the results of $T_{5}$ in the database:

* If we have, then call Algorithm 2.3

$\rightarrow$ Generating triangulations and displaying results in browsers (Figure 3.3)

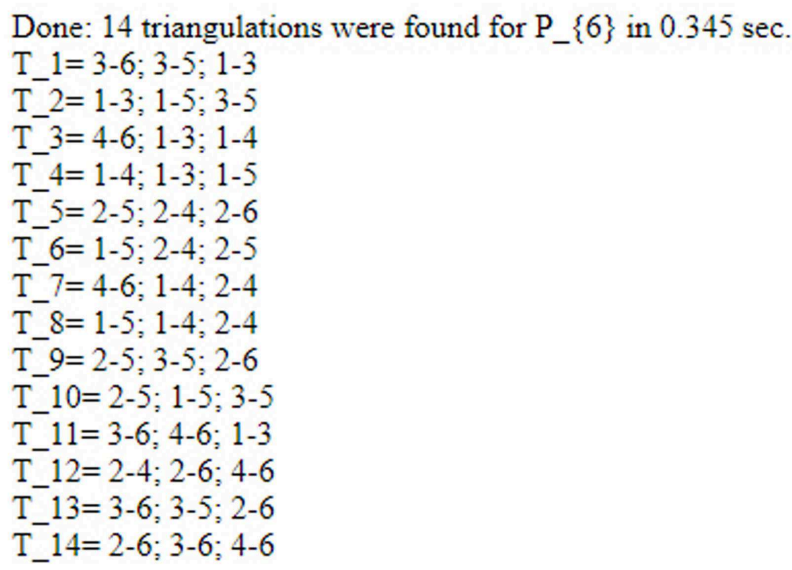

FIG. 3.3: Generating results for $T_{6}$ 


\section{Comparative analysis and experimental results}

The main idea of our implementation is to provide an appropriate client-server web application, in the free open source PHP/MySQL development environment, utilizing the minimum of resources: an internet browser and an operating system.

For a comparative analysis in presenting the advantages of web technologies, we implement an additional algorithm from the field of computer graphics (Orbiting Triangle method [8]).

Both algorithms are based on the usage of the previously generated triangulations for a polygon with a smaller number of vertices.

The execution times with respect to two criteria are presented in Table 4.1. The table column "Speedup" shows the quotient of the values contained in the previous two columns.

The testing is performed on the following configuration*: $C P U$ - Inter $(R)$ Core (TM) i5-4210U CPU@1.70GHz 2.40GHz, RAM memory 8GB, Graphics card: NVIDIA GeForce 820M.

Table 4.1: The execution times of computing triangulations (in seconds)

\begin{tabular}{r|r|rrr|rrr}
\hline$n$ & $\begin{array}{c}\text { Number of } \\
\text { triangulations }\end{array}$ & $\begin{array}{c}\text { BM in } \\
\text { generating }\end{array}$ & $\begin{array}{c}\text { BM in reading } \\
\text { from DB }\end{array}$ & Speedup & $\begin{array}{c}\text { OTM in } \\
\text { generating }\end{array}$ & $\begin{array}{c}\text { OTM in reading } \\
\text { from DB }\end{array}$ & Speedup \\
\hline 5 & 5 & 0.256 & 0.003 & $\mathbf{8 5 . 3 3}$ & 0.067 & 0.001 & $\mathbf{6 7 . 0 0}$ \\
6 & 14 & 0.345 & 0.003 & $\mathbf{1 1 5 . 0 0}$ & 0.088 & 0.001 & $\mathbf{8 8 . 0 0}$ \\
7 & 42 & 0.391 & 0.003 & $\mathbf{1 3 0 . 3 3}$ & 0.123 & 0.001 & $\mathbf{1 2 3 . 0 0}$ \\
8 & 132 & 0.457 & 0.004 & $\mathbf{1 1 4 . 2 5}$ & 0.185 & 0.002 & $\mathbf{9 2 . 5 0}$ \\
9 & 429 & 0.756 & 0.008 & $\mathbf{9 4 . 5 0}$ & 0.927 & 0.002 & $\mathbf{4 6 3 . 5 0}$ \\
10 & 1,430 & 1.606 & 0.019 & $\mathbf{8 4 . 5 3}$ & 1.524 & 0.003 & $\mathbf{5 0 8 . 0 0}$ \\
11 & 4,862 & 3.915 & 0.063 & $\mathbf{6 2 . 1 4}$ & 3.182 & 0.008 & $\mathbf{3 9 7 . 7 5}$ \\
12 & 16,796 & 26.657 & 0.461 & $\mathbf{5 7 . 8 2}$ & 10.081 & 0.024 & $\mathbf{4 2 0 . 0 4}$ \\
13 & 58,786 & 185.566 & 2.482 & $\mathbf{7 4 . 7 6}$ & 29.713 & 0.075 & $\mathbf{3 9 6 . 1 7}$ \\
14 & 208,012 & 883.726 & 6.802 & $\mathbf{1 2 9 . 9 2}$ & 121.749 & 0.248 & $\mathbf{4 9 0 . 9 2}$ \\
15 & 742,900 & $4,498.768$ & 25.697 & $\mathbf{1 7 5 . 0 7}$ & 536.326 & 0.975 & $\mathbf{5 5 0 . 0 8}$ \\
\hline
\end{tabular}

\section{Conclusion}

We implemented the Block method for convex polygon triangulation in the web environment using the open source software (PHP/MySQL). With this implementation we presented the advantages of web technologies in preforming a complex algorithm from computer graphics. The research also contributes to the manner in which an MySQL database is used for storing the obtained results and utilizing them for another calculation. As presented in the comparative analysis section, we can conclude that the advantages of using a database in performing complex algorithms are justified. This way of implementation provides a good basis for further application of the web technology in computing other algorithms. 


\section{A Important source code of the implementation}

Source code for creating a MySQL database:

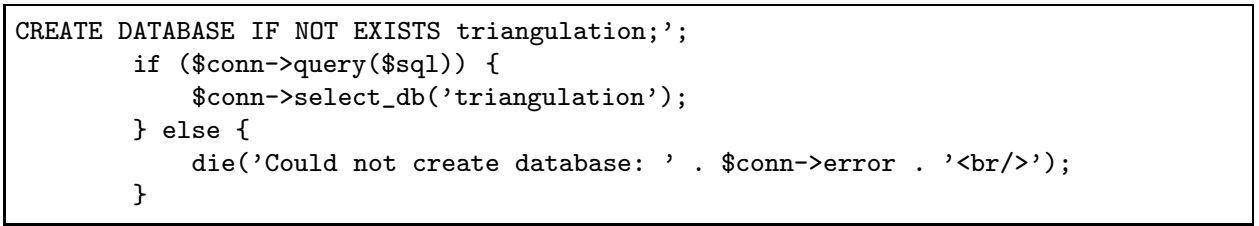

The source code for database connection:

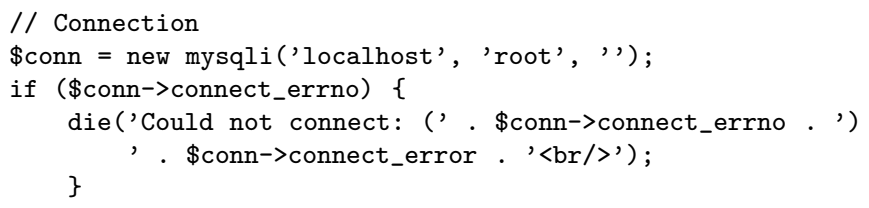

In our implementation, we use only one table for storing generated triangulations.

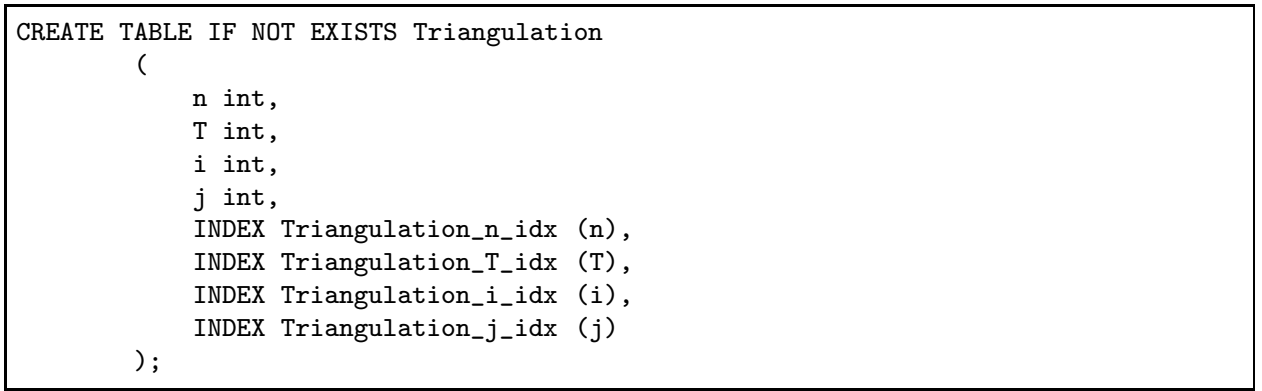

The source code of the implementation of Algorithm 2.3 - step 3:

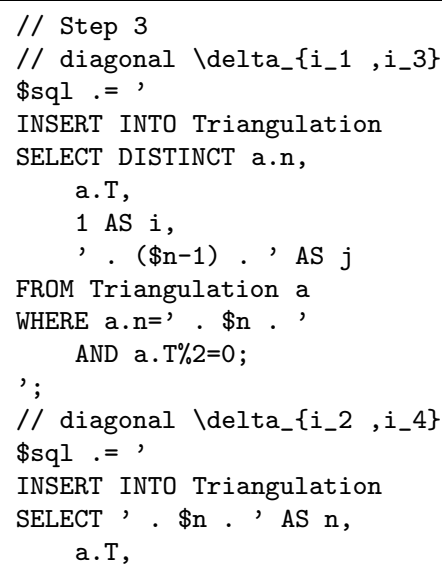




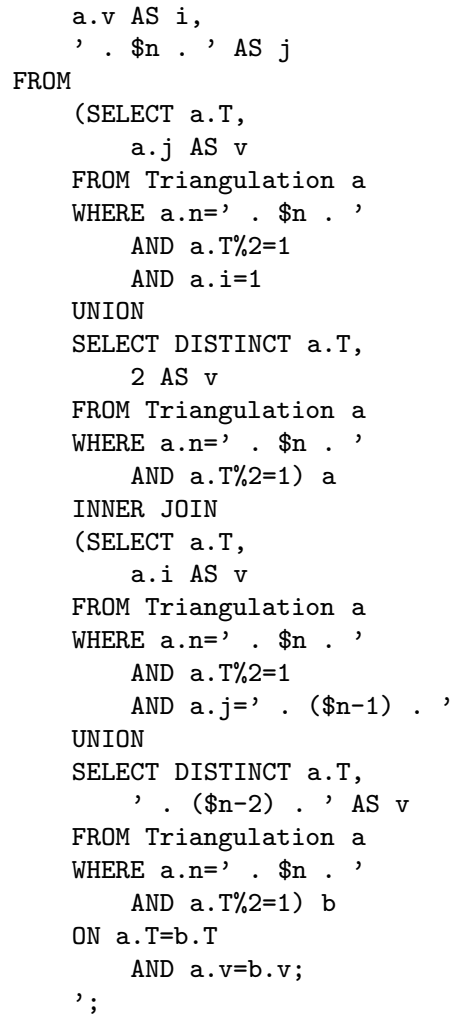

The source code of the implementation of Algorithm 2.3 - step 4:

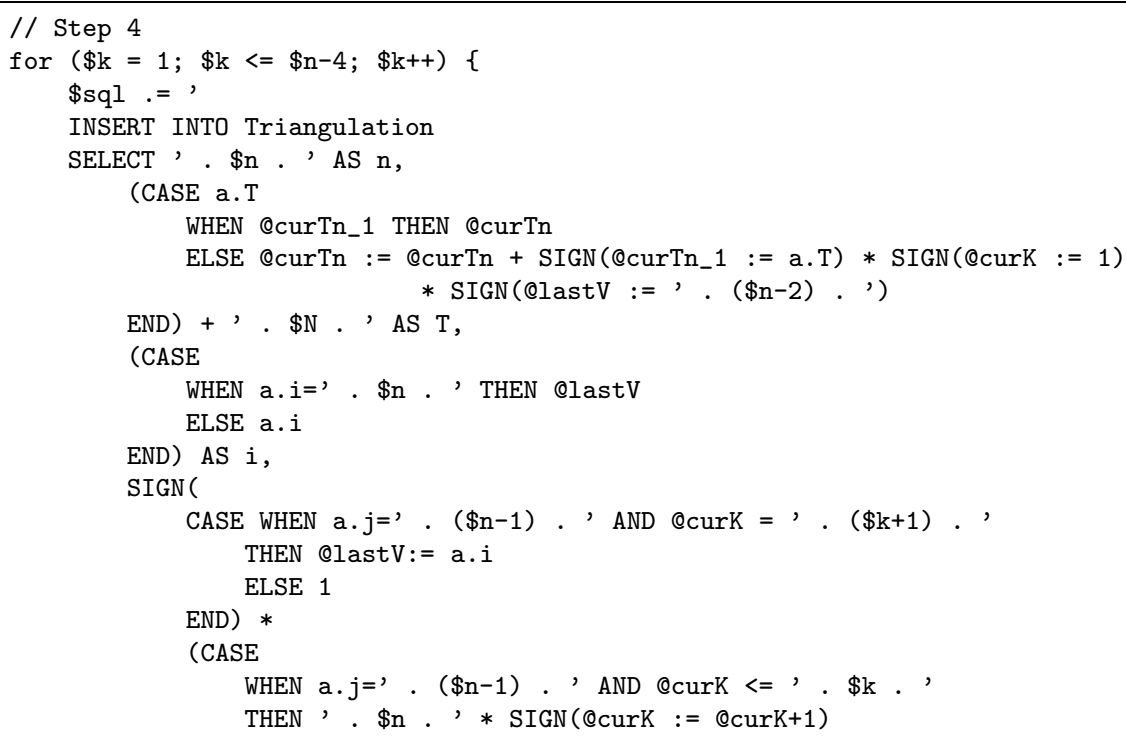


ELSE $a \cdot j$

END) AS $j$

FROM

(SELECT a.T,

a.i,

a.j

FROM Triangulation a

WHERE a. $n=$, . $(\$ n-1)$.

AND a.T IN

(SELECT a.T

FROM Triangulation a

WHERE $a \cdot n=\prime$. $(\$ n-1)$.

AND $a \cdot j=, \cdot(\$ n-1)$.

GROUP BY a.T

HAVING count (a.i)>=', .\$k . ')

UNION SELECT a.T,

, . \$n. , AS i,

'. \$n. 'AS $j$

FROM Triangulation a

WHERE $a \cdot n=\prime$. $(\$ n-1)$.

AND $a \cdot j=\prime,(\$ n-1)$.

GROUP BY a.T

HAVING $\operatorname{count}(\mathrm{a} . \mathrm{i})>=$, , $\$ \mathrm{k} .$,$) , a$

(SELECT @curTn := 0, @curTn_1 :=0, @curK := 0, @lastV $:=$, . (\$n-2).') b ORDER BY a.T,

a.i,

,

a.j;

if ( $\$$ result $=$ \$conn->query ('

SELECT a.T

FROM Triangulation a

WHERE $a . n=' \cdot(\$ n-1)$. '

AND $a \cdot j=\prime,(\$ n-1)$.

GROUP BY a.T

HAVING count (a.i)>=', \$k . ';

)) \{

$\$ \mathrm{~N}+=$ \$result->num_rows;

\$result->close();

else \{

die('Could not access Triangulation table: ' . \$conn->error . '<br/>');

\}

\}

\section{RE F E R E N C E S}

1. D. Lane, H. Williams: Web Database Applications with PHP and MySQL, 2nd Edition, O'Reilly Media, 2009.

2. M. Rahman: PHP 7 Data Structures and Algorithms: Implement Linked Lists, Stack, and Queues Using PHP, Packt Publishing, 2017. 
3. M. SaraČević, P. Stanimirović, S. Mašović, E. BiševaC: Implementation of the convex polygon triangulation algorithm, Facta Universitatis Math. Inform. 27 (2012), pp. 213-228.

4. M. Tasić, P. Stanimirović, S. Pepić: Computation of generalized inverses using Php/MySql environment, Int. J. Comput. Math. 88 (2011), pp. 2429-2446.

5. P. Krtolica, P.Stanimirović, M. Tasić, S. Pepić: Triangulation of Convex Polygon with Storage Support, Facta Universitatis, 29:2 (2014), pp. 189-208.

6. P. Stanimirović, P. Krtolica, M. Saračević, S. Mašović: Block Method for Convex Polygon Triangulation, Rom. J. Inf. Sci. Tech. 15:4 (2012), pp. 344-354.

7. R. NIXON: Learning PHP, MySQL $\&$ JavaScript, 5th Edition, O'Reilly Media, 2018.

8. S. Mašović, I. ElshaArawz, P. Stanimirović, P. Krtolica: Orbiting triangle method for convex polygon triangulation, Applicable Analysis and Discrete Mathematics, 12 (2018), pp. 439-454.

9. T. Koshy: Catalan Numbers with Applications, Oxford University Press, New York, 2009.

Sead H. Mašović

University of Niš

Faculty of Science and Mathematics

Department of Computer Science

18000 Niš, Serbia

sead.masovic@pmf.edu.rs

Muzafer H. Saračević

University of Novi Pazar

Department of Computer Science

36300 Novi Pazar, Serbia

muzafers@uninp.edu.rs

Predrag S. Stanimirović

University of Niš

Faculty of Science and Mathematics

Department of Computer Science

18000 Niš, Serbia

pecko@pmf.ni.ac.rs

Predrag V. Krtolica

University of Niš

Faculty of Science and Mathematics

Department of Computer Science

18000 Niš, Serbia

krca@pmf.ni.ac.rs 